\title{
BMJ Open Effects of a workplace exercise intervention on cardiometabolic health: study protocol for a randomised controlled trial
}

\author{
Ali Muneer Alrahma (D) , ${ }^{1,2}$ Mansoor Anwar Habib, ${ }^{3}$ Abderrahim Oulhaj (D) , \\ Tom Loney, ${ }^{4}$ Thomas Boillat, ${ }^{5}$ Syed M Shah (D) , ${ }^{1}$ Luai A Ahmed (D) , \\ Javaid Nauman (D) $1,6,7$
}

To cite: Alrahma AM, Habib MA, Oulhaj A, et al. Effects of a workplace exercise intervention on cardiometabolic health: study protocol for a randomised controlled trial. BMJ Open 2021;11:e051070. doi:10.1136/ bmjopen-2021-051070

- Prepublication history and additional supplemental material for this paper are available online. To view these files, please visit the journal online (http://dx.doi.org/10.1136/ bmjopen-2021-051070)

Received 10 March 2021 Accepted 24 September 2021

Check for updates

(c) Author(s) (or their employer(s)) 2021. Re-use permitted under CC BY-NC. No commercial re-use. See rights and permissions. Published by BMJ.

For numbered affiliations see end of article.

Correspondence to Dr Javaid Nauman; javaid.nauman@uaeu.ac.ae

\section{ABSTRACT}

Introduction The worldwide rising levels of physical inactivity especially in the United Arab Emirates (UAE) and the Eastern Mediterranean region are alarming. The UAE reports one of the highest rates of non-communicable disease mortality and insufficient physical activity (PA) is a major underlying cause. Therefore, action is required to reduce physical inactivity using evidence-based strategies. This study aimed to evaluate the efficacy of a worksite exercise intervention on cardiometabolic health in the UAE. Methods and analysis This is a protocol for a pragmatic parallel randomised controlled trial with a 1:1 allocation ratio to the intervention group and delayed intervention group. A total of 150 participants will be recruited from a semigovernment telecommunications company in Dubai (UAE) after meeting the eligibility criteria. The intervention group will receive 2 hours of exercise per week during working hours for 12 weeks (maximum 1 hour/day). The intervention group will be assigned to attend personal trainer sessions in the workplace gym throughout the intervention period. After the intervention is completed, the delayed intervention group will also receive 2 hours of exercise time per week from working hours for 4 weeks. The main outcome measure is the change in the cardiometabolic risk components, that is, systolic or diastolic blood pressure, waist circumference, glycated haemoglobin, fasting plasma glucose, lowdensity lipoprotein cholesterol from baseline to the end of the intervention. The secondary outcome is to examine whether the workplace exercise intervention improves PA levels 4 weeks postintervention.

Ethics and dissemination The study has been approved by the Dubai Scientific Research Ethics Committee (DSREC-SR-08/2019_02). The results will be disseminated as follows: at various national and international scientific conferences; as part of a PhD thesis in Public Health at the College of Medicine and Health Sciences, UAE University; and in a manuscript submitted to a peer-reviewed journal. Trial registration number NCT04403789.

\section{INTRODUCTION}

Overwhelming evidence shows that insufficient physical activity (PA) is associated with many chronic diseases such as circulatory

\section{Strengths and limitations of this study}

- This study is a prospective, single-blind randomised controlled trial which is the most robust study design to answer the research question.

- Adequately powered study using intention-to-treat analysis will provide strong evidence for the efficacy of worksite exercise interventions.

- Personal trainers will supervise the exercise sessions to ensure intervention compliance.

- The intervention will be aligned with the European Network for Workplace Health Promotion recommendations, and time allocation to participants will be during their working hours to complete structured exercise sessions.

- There is a risk of contamination between the two groups due to the nature of the study, and the generalisability of the findings might be limited to similar populations and workplace settings.

diseases, depressive disorders, musculoskeletal diseases, diabetes, breast cancer and diseases of the digestive system, and contributes to a financial burden on health systems and on individuals worldwide. ${ }^{1-7}$ On the contrary, regular PA may serve as an effective and cost-effective non-pharmacological therapy that improves health by reducing the prevalence of different comorbid conditions, including hypertension, overweight and obesity, as well as lowering the risk of death from cardiovascular disease (CVD) and improving the quality of life and mental health. ${ }^{589}$

\section{Cardiometabolic risk factors and PA}

Cardiometabolic risk factors (CRFs) are a group of risk factors that increase the risk of chronic non-communicable diseases (eg, diabetes and CVDs). ${ }^{10} 11$ CRF components include but are not limited to waist circumference, elevated systolic blood pressure, 
elevated low-density lipoprotein (LDL) cholesterol, and pre-diabetes. ${ }^{10-14} \mathrm{PA}$ is vital in improving the outcome of these risk factors. The findings of interventional studies have clearly shown a beneficial effect of PA in clinical or community settings. ${ }^{15-17}$ However, there is limited evidence for the effectiveness of PA interventions in worksite settings where individuals spend the majority of their waking hours.

\section{Physical inactivity in the workplace}

One of the most prominent factors affecting PA is the environment that surrounds the individual..$^{18}$ An environment could include the workplace, school setting, public places (beaches, neighbourhoods, and parks), sports facilities, family, and community centres. ${ }^{19}$ The 2018-2030 Global Action Plan on Physical Activity by WHO considers the workplace environment as an important arena that could promote PA. ${ }^{19}$ In addition, WHO states that the workplace is considered an ideal setting to provide planned and structured activities for employees to improve their overall health. ${ }^{20}$

A systematic review relating to workplace PA interventions included four narrative reviews and one metaanalysis. ${ }^{16}$ The findings showed that the majority of studies included in the narrative reviews were of poor methodological quality, ${ }^{21-24}$ showed inconclusive results, ${ }^{22}$ or focused on analysing sitting time instead of low levels of PA. ${ }^{24}$ The meta-analysis included PA interventions with various study designs, showed the lack of randomised clinical trials in the workplaces, and reported that objective measurements were rare among the studies included. In addition, the meta-analysis found that only $27 \%$ of the included studies had supervised exercise sessions for the participants. ${ }^{25}$ Furthermore, a recent worksite intervention investigated daily walking time between employees that used treadmill workstations (intervention group) and sit-stand desk. ${ }^{26}$ The study reported that although the primary goal was not met, there was a significant increase in daily walking time in the intervention group (increase of $18 \mathrm{~min}$ from baseline to 13 months). ${ }^{26}$ However, data remain scarce for PA intervention studies in the workplace, and there is a need for more evidence-based interventions in the workplace that examine their effect on metabolic risk factors. ${ }^{21}$

The European Network for Workplace Health Promotion (ENWHP) recommends a set of criteria for the promotion of $\mathrm{PA}$ in the workplace. ${ }^{27}$ The criteria include implementing approaches that encourage PA during working hours, weekends, and non-working hours. In addition, the ENWHP recommends providing easily accessible PA facilities and programmes in the workplace or at least in external sports facilities. The final recommendation is to raise the awareness of employees through extensive information about the importance of PA. ${ }^{27}$

The alarming rising levels of physical inactivity in the Eastern Mediterranean region (43\%) and in the United Arab Emirates (UAE) (38\%) are comparable with the global levels of insufficient PA $(31 \%) .{ }^{28} 29$ The
UAE government is advocating the importance of PA and a healthy lifestyle. For instance, in the National Agenda Vision 2021, the UAE aims to promote healthy and long life not only through health services but also through prevention and awareness of healthy lifestyle behaviours. ${ }^{30}$ In addition, the National Agenda aims to prevent disease through early interventions that lead to behaviour change and consequently improves general health status and quality of life. ${ }^{30}$ Therefore, we aim to conduct a randomised clinical study following the ENWHP recommendations to examine the effects of a workplace exercise intervention on cardio-metabolic health outcomes.

\section{OBJECTIVES}

\section{Primary objective}

The primary objective of this study was to evaluate the effect of a 12-week workplace structured exercise intervention on cardiometabolic risk factors.

\section{Secondary objective}

To determine whether the workplace exercise intervention will improve PA levels 4 weeks postintervention.

\section{METHODS AND ANALYSIS \\ Trial design}

The study is considered a pragmatic parallel randomised controlled trial with a 1:1 allocation ratio to the intervention group and delayed intervention group. Participants' enrolment will start on 28 March 2021, and the study is expected to end on 30 November 2021.

\section{Study setting}

The intervention will be conducted in the headquarter building of a semigovernment telecommunications company in Dubai, UAE (Du Emirates Integrated Telecommunications Company, PJSC). The headquarters building includes a gym, a health centre and a swimming pool (6.14 $\mathrm{m}$ in length and $4.10 \mathrm{~m}$ in width). The gym is dedicated for Du staff members. It includes a wide range of exercise equipment and facilities such as free weights, weight machines, rowing machines, treadmills, cycle ergometers and space for group classes. All structured exercise intervention sessions will be conducted at the workplace gym facility, which is the only study site. The health centre at Du headquarters is also dedicated for the company's staff. It provides both preventive and curative services. Preventive services include screening, vaccination and health education. Curative services include management of all acute and chronic illnesses from consultation to blood tests and writing prescriptions (licensed family medicine clinic).

\section{Patient and public involvement}

The public or participants were not involved in designing, conducting, reporting or dissemination plans of the study protocol. 


\section{Recruitment}

An email invitation to attend an information session will be sent to all the company's employees in Dubai, UAE. The invitation will emphasise that participation is voluntary and that they can withdraw at any time without giving any reason. The information session will discuss the study details (eg, eligibility criteria) and advice for increasing PA. All employees who wish to participate will need to sign a consent form. Eligible participants will have an appointment in the worksite health centre to complete the required baseline health measurements.

Furthermore, we do not plan to offer any monetary compensation to participants. However, if recruitment rates are lower than expected, we will pilot whether offering free (non-monetary) gifts, certificates or first aid kits improves response rates.

\section{Eligibility criteria}

The eligibility criteria for participants to join the workplace intervention include (inclusion criteria) the following:

1. The participant must be an employee in the company and have a waist circumference of $\geq 94 \mathrm{~cm}(\geq 90 \mathrm{~cm}$ for South and East Asians) for men and $\geq 80 \mathrm{~cm}$ for women.

2. Age $18-59$ years old.

3. Availability for the study duration.

4. Willingness of the participant to commit to the intervention until the end.

5. Signed written consent to participate.

Exclusion criteria include

1. Severe injury in the joints or the back or any medical condition that would prevent them from exercising, or the participant is advised not to exercise by a doctor.

2. Pregnancy.

3. Any planned major surgical procedures during the intervention period.

4. Self-reported CVD, lung disease or cancer.

5. Currently participating in another health promotion programme.

\section{Intervention}

The intervention duration will be 12 weeks, and it will provide an exercise time of 2 hours/week during the normal working hours. The 2 hours should be used in 2 separate days per week (a maximum of 1 hour/day), either in the middle or at the end of the working hours. The intervention duration was chosen based on recommendations from previous studies that showed 12 weeks were adequate to observe significant changes in the selected cardiometabolic outcomes. ${ }^{16} 31$ The exercise sessions will be conducted under the supervision of certified exercise trainers in the workplace gym. The exercise types and durations are all based on the recommendations by the American College of Sports Medicine ${ }^{32}$ and will ensure that all major muscle groups are targeted. For instance, each 1 hour session will be conducted at a moderate to high intensity, and starts with 5 min warm-up exercises, then $50 \mathrm{~min}$ resistance and aerobic exercises, and finally with $5 \mathrm{~min}$ cool-down exercises. The resistance and aerobic exercises are performed in $7-10 \mathrm{~min}$ bouts. ${ }^{32}$ The delayed intervention group will be asked to maintain their usual lifestyle. However, after the completion of the intervention period, the delayed intervention group will also receive 2 hours of exercise time per week from working hours for 4 weeks.

\section{Comparators}

Intervention group

The duration of the intervention is 12 weeks, and certified exercise trainers will supervise the group sessions during working hours.

\section{Delayed intervention group (active comparator)}

The duration of the delayed intervention is 4 weeks and starts after the intervention group finishes the 12-week intervention. Exercise hours during the scheduled workday will be granted to this group; however, they will not be supervised by a certified trainer.

The main difference between the intervention group and the delayed-intervention group is the timing and duration of the intervention. In addition, the certified trainers will only be available for the intervention group. The purpose of the delayed intervention group is to encourage participants to participate in the study regardless of their group allocation.

\section{Sample size calculation}

Previous studies relating to PA and cardiometabolic risk factors used $80 \%-91 \%$ power and effect sizes ranging between 0.51 and 1.82 to find a significant difference between groups. ${ }^{33-36}$ There are various reasons for choosing these articles for sample size calculations. For example, one study used a 12-week PA intervention that involved healthy adult participants. ${ }^{36}$ In addition, the articles cited were concerned with the effect of exercise on specific metabolic risk factors. These metabolic risk factors were waist circumference, systolic blood pressure, fasting glucose and lipid profiles. ${ }^{33-37}$ For the present study, we estimated that 124 participants will be required at $80 \%$ power. A further $20 \%$ more participants will be added because it is expected that participants might drop out during the intervention. Therefore, we will recruit a total of 150 participants.

\section{Allocation}

Sequence generation

Eligible participants will be randomised 1:1 to intervention group $(\mathrm{n}=75)$ and delayed intervention group $(\mathrm{n}=75)$ via random computerised allocation to minimise selection bias. Figure 1 shows the allocation of participants into two arms.

\section{Allocation concealment mechanism}

In order to provide a balance between the trial arms for sex and age groups, participants will be allocated remotely by concealed minimisation. A biostatistician will 


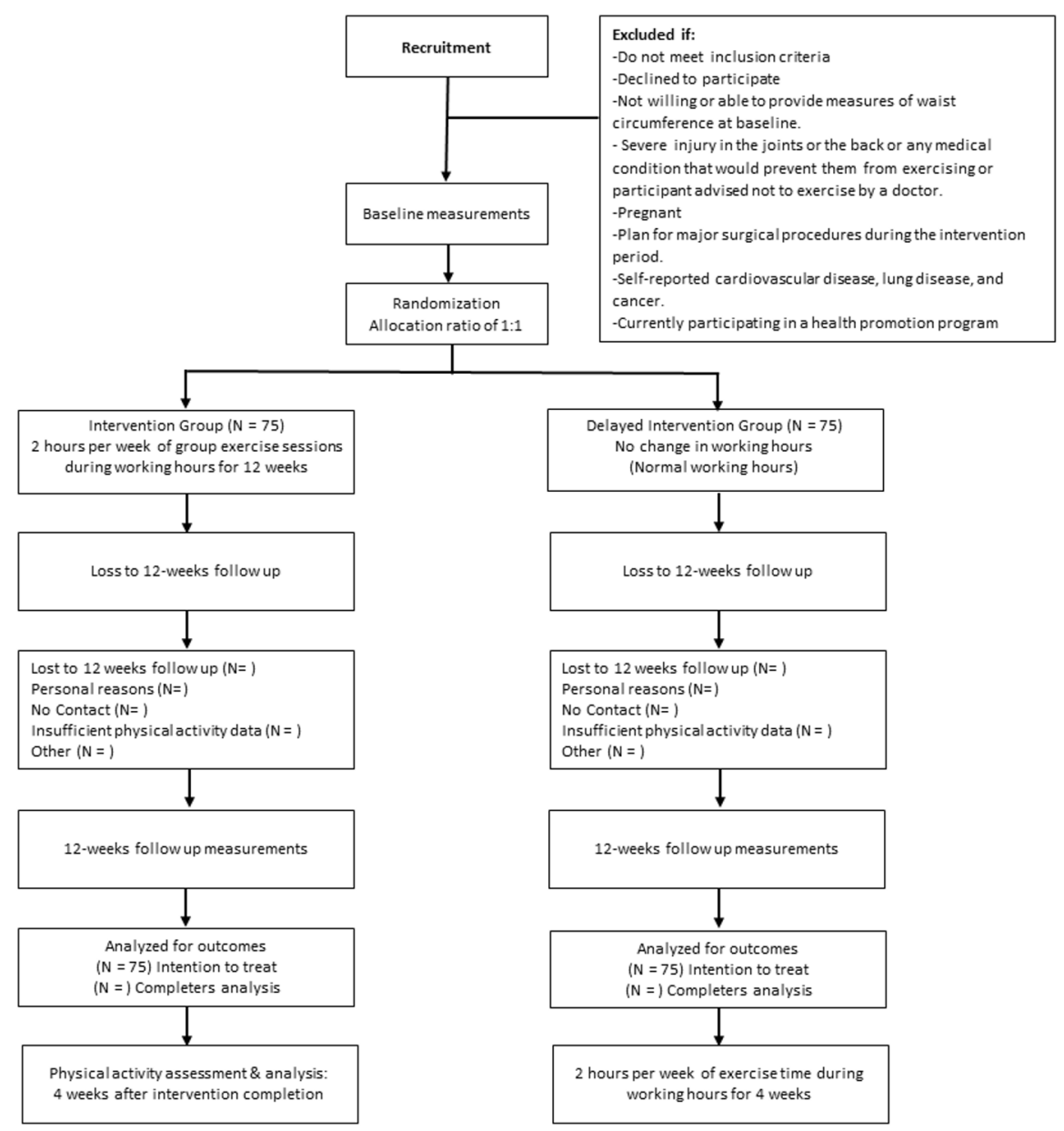

Figure 1 Consolidated Standards of Reporting Trials flow diagram.

be responsible for this task, and they will not be related to any part of the study.

\section{Blinding}

Single blinding will be used to minimise measurement and performance bias from the assessors. Participants will be strongly encouraged not to disclose their allocation status during the health measurement assessments. In terms of unblinding participants, it will not be required for this study.

\section{Outcomes}

The main outcome in the study includes cardiometabolic risk components: waist circumference, glycated haemoglobin (HbA1c), fasting blood glucose, LDL cholesterol, and blood pressure. The secondary outcome will be the change in subjectively and objectively measured PA levels. The cardio-metabolic risk components will be measured for both groups before and after the intervention. However, 4 weeks after completing the intervention, only PA levels will be assessed subjectively and objectively for the third time for the intervention group.

\section{Measurements}

The health outcomes will be measured in the workplace's health centre (eg, blood samples, blood pressure, waist circumference, height, weight and questionnaires). A butterfly needle will be used to collect blood samples for HbAlc, fasting blood glucose, and LDL cholesterol after 12 hours of fasting. The drawn blood samples will then be stored in a $-20^{\circ} \mathrm{C}$ or colder freezer, and sent to analysis (Automated HbA1c analyser FORA A1C100, UAE; Glucose and Cholesterol metre SD LipidoCare, South Korea). Resting diastolic and systolic blood pressure (Omron, Japan) will be measured after the participant sits for at least $5 \mathrm{~min}$ on a chair with back support. Waist circumference will be measured in centimetres using a measurement tape. The participant must be standing when the tape is placed above the hipbones. The measurement will be taken when the tape is not compressed on the skin and after breathing out. Body mass in kilogram and height in centimetre will be measured with a body composition machine (Inbody 230, Korea; with built-in height measurement tool BSM370, Korea). The participant will need to empty the pockets and remove the shoes for the body composition measurements.

All participants will also wear the tri-axial accelerometer (AX3 Axivity, UK) on the dominant wrist (hand used to write) for seven consecutive days (baseline and postintervention), similar to previous studies. ${ }^{38} 39$ The accelerometer devices will be configured to capture 


\begin{tabular}{ll}
$\begin{array}{l}\text { Table } 1 \text { Measurement criteria for cardiometabolic risk } \\
\text { factor components }\end{array}$ & Criteria \\
\hline Health outcome & $\begin{array}{l}\text { Systolic blood } \\
\text { pressure } \geq 130 . \\
\text { Diastolic blood } \\
\text { pressure } \geq 80 .{ }^{1145}\end{array}$ \\
\hline High blood pressure (mm Hg) & $>102$ (men). \\
& $>>88$ (women). ${ }^{13}$ \\
$\begin{array}{l}\text { Waist circumference (cm) } \\
\begin{array}{l}\text { Elevated LDL cholesterol (mg/ } \\
\text { dL) }\end{array}\end{array}$ \\
$\begin{array}{l}\text { Pre-diabetes } \\
\text { HbA1c (glycated } \\
\text { haemoglobin \%) }\end{array}$ \\
$\begin{array}{l}\text { Fasting plasma glucose } \\
\text { (mg/dL) }\end{array}$ & $100-125^{11}$ \\
\hline
\end{tabular}

HbA1c, glycated haemoglobin; LDL, low-density lipoprotein.

three-dimensional acceleration at $100 \mathrm{~Hz}$ with a dynamic range of $\pm 8 \mathrm{~g}$. The devices will be programmed to record data at the prespecified start and finish times. The primary and secondary health outcomes and their criteria are shown in tables 1 and 2.

The validated WHO-5 Well-Being Index questionnaire, as well as other questionnaires that measure the frequency of food consumption, eating habits and PA (International Physical Activity Questionnaire (IPAQ)) will also be used ${ }^{40-42}$ (online supplemental material).

\section{Trial registration data set}

The trial registration data set as per the SPIRIT guideline recommendations is found in the online supplemental material document.

\section{Statistical methods}

The main outcome analyses will be conducted according to the 'intention-to-treat' principle. For comparison between intervention and delayed intervention arms of the 12-week change in outcomes, we plan to use analysis of covariance model, with baseline values as the covariate to control for chance imbalances at baseline. Age-specific and sex-specific interaction analyses will be conducted. We will adopt a linear mixed model approach to provide the mean intervention effect and quantification of individual differences in response to the intervention. Accepted regression modelling methods will be used to explore the intervention effects on our secondary objective.

\section{Data analysis}

The clinical significance of the intervention effect will be elaborated with magnitude-based inferences, CIs and confidence levels. ${ }^{43}$ Analyses and reporting will be in line with Consolidated Standards of Reporting Trials guidelines, with all analyses being on an intention-to-treat principle, regardless of intervention compliance. Multiple imputation techniques will assess the sensitivity of the analyses to the missing at random assumption. Significance tests at $5 \%$, either t-test or $\chi^{2}$ tests, will be used to compare those with complete data and those who have missing outcomes.

\section{Accelerometer data processing and analysis}

Raw accelerometry data will be calibrated to $1 \mathrm{~g}$ of local gravity and filtered to eliminate machine noise using a fourthorder Butterworth low-pass filter (set at a cut-off frequency of $20 \mathrm{~Hz}) .{ }^{38}{ }^{39}$ Euclidean norm minus one (ENMO) is used to calculate the vector magnitude of the acceleration axes ( $x, y$ and $\mathrm{z}$ ) minus one gravitational unit ( $1 \mathrm{~g}$ ) (any negative values will be truncated to zero). ${ }^{38}{ }^{39}$ Non-wear time is identified as time periods of at least $60 \mathrm{~min}$, where all three-dimensional axes have an SD of less than $13 \mathrm{mg}$. In addition, moderateto-vigorous PA is defined as ENMO values more than 125 mg and are expressed as minutes per day. ${ }^{38}{ }^{39}$ Participants with wear time of less than 72 hours per ENMO of $500 \mathrm{mg}$ or more will be excluded from the analysis. ${ }^{38}{ }^{39}$ Lastly, the Open Movement software will be used to analyse the acceleromtry data.

Table 2 Measurement criteria for PA assessed by questionnaires

\begin{tabular}{ll}
\hline Health outcome & Criteria \\
\hline PA levels by questionnaire & Category 1: low \\
& This is the lowest level of physical activity. Those individuals who do not meet the criteria for \\
& category 2 or 3 are considered low/inactive. \\
& Category $2:$ moderate \\
& Any one of the following criteria: \\
& 3 or more days of vigorous-intensity activity of at least 20 min/day. \\
& 5 or more days of moderate-intensity activity or walking of at least 30 min/day. \\
& 5 or more days of any combination of walking, moderate-intensity or vigorous-intensity \\
& activities achieving a minimum of at least 600 (MET)-min/week. \\
& Category $3:$ high \\
& Vigne of the following criteria: \\
& 7 or more days of any combination of walking or moderate-intensity or vigorous-intensity \\
& activities, achieving a minimum of at least 3000 MET-min/week.
\end{tabular}

MET, metabolic equivalent of task; PA, physical activity. 
Criteria for discontinuing or modifying allocated interventions

The main risks of participation are direct physical harm and breaches of privacy or confidentiality. The risks of direct physical harm are minimal. All clinical staff will receive full training on all of the study procedures, and all measurements will be performed by trained staff/nurses. The exercise sessions will be conducted under the supervision of certified exercise trainers to ensure proper exercise techniques and therefore reduce the risk of any potential injury. Participants' health information will be protected by restricted access and using deidentified labelling, and the use of deidentified data sets for analysis.

Each participant has the right to withdraw from the study at any time without giving any reason. Should participants wish to withdraw from the study, they are advised in the participant information leaflet to contact the study coordinator. Should they wish for their samples and data collected to be destroyed, this will be done in a prompt and secure manner, and a message will be sent to the person to confirm this. The reason for withdrawal, if given, will be recorded in the database.

\section{Strategies to improve adherence to intervention protocols}

The completion of the following tasks ensures adherence and monitoring of the intervention protocols:

- Inviting all employees to the information session and recruiting eligible participants.

- Randomly allocating participants to the intervention and delayed intervention group.

- Collecting and analysing blood samples during preintervention and postintervention periods.

- PA levels recorded through IPAQ and accelerometer.

- Recording participant's attendance to personal trainer sessions.

Plans to promote participant retention and complete follow-up During the intervention, participants allocated in the intervention group will receive exercise time of 2 hours per week. The 2 hour exercise session will be conducted under the supervision of a certified trainer in the gym workplace. In addition, after the intervention is completed, the delayed intervention group will also receive 2 hours of exercise time per week from working hours for 4 weeks. These 2 hours of exercise during the workplace hours will promote participant retention and complete follow-up.

\section{Data management}

The study investigators will ensure that the participants' anonymity is maintained. All data will be stored securely in an electronic database. Participants will be assigned a unique research number, and no individually identifiable information will be included in the database. The research number linked to personally identifying information will be maintained in a secure server physically separate from the electronic database, which will be accessible only by designated staff. This secure linkage will allow for the subsequent addition of new information while maintaining no personal identifiers in the research database. Questionnaire responses and physical measures will be maintained under the unique research number.

Furthermore, we will also develop a detailed coding scheme and coding checking protocol, crosstabulation, negative case analysis, and respondent validation to enhance dependability and trustworthiness.

\section{Data monitoring}

The data monitoring committtee consists of the principal investigator and $\mathrm{PhD}$ candidate who will have access to the final trial data set. The steering committee has the authority to stop or modify the study.

\section{Process evaluation}

Realistic evaluation will be applied at the end of the study to evaluate the process and implementation elements of the study. A questionnaire will be sent to all participants after completing the study requirements. The questionnaire as presented in the online supplemental material includes major components of realistic evaluation such as context, mechanisms, facilitators and inhibitors of the intervention, and outcomes. ${ }^{44}$

\section{Participant timeline}

A schematic for the time schedule of enrolment, interventions and assessments is presented in figure 2 .

\section{Potential harms}

All clinical staff will receive full training on all of the study procedures, and all measurements will be performed by trained staff/nurses. The risks of direct physical harm are minimal. However, in case of any harm, it will be reported to the trial management committee.

\section{ETHICS AND DISSEMINATION}

\section{Research ethics approval}

The investigators will ensure that this study is conducted in accordance with the principles of the Declaration of Helsinki, and the conduct is in full conformity with relevant regulations and with the ICH Guidelines for Good Clinical Practice (CPMP/ICH/135/95) July 1996. Any research conducted in the emirate of Dubai must be submitted to the Dubai Scientific Research Ethics Committee (DSREC) in Dubai Health Authority. Therefore, the research protocol, informed consent form, participant information sheet, questionnaires and any proposed advertising material were submitted to the DSREC. The study received an ethical approval from this committee with the reference number DSRECSR-08/2019_02. In case of any future amendments to the protocol, the DSREC will be informed.

\section{Dissemination policy}

Regardless of the magnitude or direction of study findings, the results will be published in a peer-reviewed journal. 


\begin{tabular}{|c|c|c|c|c|c|c|}
\hline & \multicolumn{6}{|l|}{ STUDY PERIOD } \\
\hline & Pre-study consent & \begin{tabular}{|l|} 
Pre-study baseline \\
\end{tabular} & $\begin{array}{c}\text { 12-week } \\
\text { Intervention }\end{array}$ & $\begin{array}{c}\text { Post-study outcomes } \\
1\end{array}$ & $\begin{array}{l}\text { 4-weekDelaved } \\
\text { interentionP Post- } \\
\text { study }\end{array}$ & $\begin{array}{c}\text { Post-study outcomes } \\
2\end{array}$ \\
\hline TIMEPOINT** & $\begin{array}{c}\text { (Mar.2021) } \\
t_{1}\end{array}$ & $\begin{array}{c}\text { (April.2021) } \\
0\end{array}$ & $\begin{array}{c}\text { (May-Aug. 2021) } \\
t_{t}\end{array}$ & \begin{tabular}{|c|} 
(Aug. - Sep.2021) \\
$t_{\text {t }}$
\end{tabular} & $\begin{array}{c}\text { (Sep. 2021) } \\
t_{3}\end{array}$ & $\begin{array}{c}\text { (Sep.-Nov. 2021) } \\
\mathrm{t}_{4}\end{array}$ \\
\hline \multicolumn{7}{|l|}{ ENROLMENT: } \\
\hline Eligibility screen & $\mathrm{x}$ & & & & & \\
\hline Informed consent & $\mathrm{x}$ & & & & & \\
\hline $\begin{array}{l}\text { Appointmentin } \\
\text { health center }\end{array}$ & $\mathrm{x}$ & & & $\mathrm{x}$ & & \\
\hline Allocation & & $\mathrm{x}$ & & & & \\
\hline Randomization & & $\mathrm{x}$ & & & & \\
\hline \multicolumn{7}{|l|}{ INTERVENTIONS: } \\
\hline Interventiongroup & $\mathrm{x}$ & & & $\mathrm{x}$ & & $\mathrm{x}$ \\
\hline $\begin{array}{l}\text { Delayed Intervention } \\
\text { group }\end{array}$ & $\mathrm{x}$ & & & $\mathrm{x}$ & & \\
\hline \multicolumn{7}{|l|}{ ASSESSMENTS: } \\
\hline $\begin{array}{l}\text { Blood pressure } \\
(\mathrm{mmHg})\end{array}$ & $\mathrm{x}$ & & & $\mathrm{x}$ & & \\
\hline $\begin{array}{l}\text { Waist circumference } \\
(\mathrm{cm})\end{array}$ & $\mathrm{x}$ & & & $\mathrm{x}$ & & \\
\hline $\begin{array}{l}\text { LDL-cholesterol } \\
(\mathrm{mg} / \mathrm{dL}) \text { la }\end{array}$ & $\mathrm{x}$ & & & $\mathrm{x}$ & & \\
\hline $\begin{array}{l}\text { HbAlc(Glveated } \\
\text { Hemoglobin \%) }\end{array}$ & $\mathrm{x}$ & & & $\mathrm{x}$ & & \\
\hline $\begin{array}{l}\text { Fasting plasma } \\
\text { glucose (mg'dL) }\end{array}$ & $\mathrm{x}$ & & & $\mathrm{x}$ & & \\
\hline $\begin{array}{l}\text { International } \\
\text { Physical Activity } \\
\text { Questionnaire }\end{array}$ & $\mathrm{x}$ & & & $\mathrm{x}$ & & $\mathrm{x}$ \\
\hline $\begin{array}{l}\text { PhysicalActivity } \\
\text { Measurement(AX3) }\end{array}$ & $\mathrm{x}$ & & & $\mathrm{x}$ & & $\mathrm{x}$ \\
\hline RealistEvaluation & & & & & & $\mathrm{x}$ \\
\hline
\end{tabular}

Figure 2 Schematic diagram for schedule of enrolment, interventions, and assessments. HbA1c, glycated haemoglobin; LDL, low-density lipoprotein.

\section{Informed consent}

Written versions of the participant information leaflet and informed consent will be presented to the participants detailing no less than the exact nature of the study, what it will involve for the participant, the implications and constraints of the protocol, and the known side effects and any risks involved in taking part. It will be clearly stated that the participant is free to withdraw from the study at any time for any reason without prejudice to future care, and with no obligation to give the reason for withdrawal. The participant will be allowed as much time as wished to consider the information, and the opportunity to question the investigator or other independent parties to decide whether they will participate in the study. Written informed consent will then be obtained by means of participant-dated signature and dated signature of the person who presented and obtained the informed consent. The person who obtained the consent must be suitably qualified and experienced and has been authorised to do so by the principal investigator. A copy of the signed informed consent will be given to the participant.

\section{Confidentiality}

Patient names and identifying numbers (eg, medical record number) will be removed from each record and replaced by a unique study number. Data will be stored on an external hard drive, which will be kept in a locked office.

\section{Ancillary and post-trial care}

It is unlikely that anything serious which requires ancillary care will occur during the study. However, if the participant is harmed by taking part in this research project, there are no special compensation arrangements. If the participant is harmed due to someone's negligence, or have any concerns about any aspect of the way they have been approached or treated during the course of this study, they will be provided with the the contact details of the DSREC. In addition, if the participants wish to make a complaint or have any feedback, they will be able to contact the research team.

\section{Authorship eligibility guidelines}

The International Committee of Medical Journal authorship criteria will be used to ensure that there are no ghost authors in this study.

\section{Author affiliations}

${ }^{1}$ Institute of Public Health, College of Medicine and Health Sciences, United Arab Emirates University, Al-Ain, Abu Dhabi, UAE

${ }^{2}$ Department of Public Health Protection, Dubai Health Authority, Dubai, UAE

${ }^{3}$ Department of Sustainability and Wellbeing, Emirates Integrated

Telecommunications Company, Dubai, UAE

${ }^{4}$ College of Medicine, Mohammed Bin Rashid University of Medicine and Health Sciences, Dubai, UAE

${ }^{5}$ Design Lab, College of Medicine, Mohammed Bin Rashid University of Medicine and Health Sciences, Dubai, UAE

${ }^{6}$ Department of Circulation and Medical Imaging, Faculty of Medicine and Health Sciences, Norwegian University of Science and Technology, Trondheim, Norway ${ }^{7}$ Healthy Living for Pandemic Event Protection (HL - PIVOT) Netwrok, Chicago, Illinois, USA 
Contributors AMA and JN conceptualised the study and developed the study design with other coinvestigators. MAH, A0, TL, TB, SMS and LAA contributed to the development of the study design. $\mathrm{AO}$ and $\mathrm{TL}$ provided statistical expertise in clinical trial design. All authors provided edits and critiqued the manuscript for intellectual content.

Funding This work was supported by United Arab Emirates University (grant number 31M466).

Competing interests None declared.

Patient consent for publication Not applicable.

Provenance and peer review Not commissioned; externally peer reviewed.

Supplemental material This content has been supplied by the author(s). It has not been vetted by BMJ Publishing Group Limited (BMJ) and may not have been peer-reviewed. Any opinions or recommendations discussed are solely those of the author(s) and are not endorsed by BMJ. BMJ disclaims all liability and responsibility arising from any reliance placed on the content. Where the content includes any translated material, BMJ does not warrant the accuracy and reliability of the translations (including but not limited to local regulations, clinical guidelines, terminology, drug names and drug dosages), and is not responsible for any error and/or omissions arising from translation and adaptation or otherwise.

Open access This is an open access article distributed in accordance with the Creative Commons Attribution Non Commercial (CC BY-NC 4.0) license, which permits others to distribute, remix, adapt, build upon this work non-commercially, and license their derivative works on different terms, provided the original work is properly cited, appropriate credit is given, any changes made indicated, and the use is non-commercial. See: http://creativecommons.org/licenses/by-nc/4.0/.

\section{ORCID iDs}

Ali Muneer Alrahma http://orcid.org/0000-0003-2813-9795

Abderrahim Oulhaj http://orcid.org/0000-0002-5330-904X

Syed M Shah http://orcid.org/0000-0002-0956-465X

Luai A Ahmed http://orcid.org/0000-0001-5292-8212

Javaid Nauman http://orcid.org/0000-0003-1347-2448

\section{REFERENCES}

$1 \mathrm{Kyu} \mathrm{HH}$, Bachman VF, Alexander LT, et al. Physical activity and risk of breast cancer, colon cancer, diabetes, ischemic heart disease, and ischemic stroke events: systematic review and dose-response meta-analysis for the global burden of disease study 2013. BMJ 2016;354:i3857.

2 Virtanen M, Ervasti J, Head J, et al. Lifestyle factors and risk of sickness absence from work: a multicohort study. Lancet Public Health 2018;3:e545-54.

3 Ding D, Lawson KD, Kolbe-Alexander TL, et al. The economic burden of physical inactivity: a global analysis of major non-communicable diseases. Lancet 2016;388:1311-24.

4 Das P, Horton R. Physical activity-time to take it seriously and regularly. The Lancet 2016;388:1254-5.

5 Piercy KL, Troiano RP, Ballard RM, et al. The physical activity guidelines for Americans. JAMA 2018;320:2020-8.

6 Wisloff U, Lavie CJ. Taking physical activity, exercise, and fitness to a higher level. Prog Cardiovasc Dis 2017;60:1-2.

$7 \mathrm{WHO}$. Who guidelines on physical activity and sedentary behaviour, 2020. Available: https://www.who.int/publications-detail-redirect/ 9789240015128 [Accessed 7 Dec 2020].

8 Fletcher GF, Landolfo C, Niebauer J, et al. Promoting physical activity and exercise: JACC health promotion series. J Am Coll Cardiol 2018;72:1622-39.

9 Kraus WE, Bittner V, Appel L, Kraus William E, Vera B, Lawrence A, et al. The National physical activity plan: a call to action from the American heart association. Circulation 2015;131:1932-40.

10 Cannon CP. Cardiovascular disease and modifiable cardiometabolic risk factors. Clin Cornerstone 2007;8:11-28.

11 Nichols GA, Horberg M, Koebnick C, et al. Cardiometabolic risk factors among 1.3 million adults with overweight or obesity, but not diabetes, in 10 geographically diverse regions of the United States, 2012-2013. Prev Chronic Dis 2017;14:E22.

12 Grundy Scott M, Stone Neil J, Bailey Alison L. 2018 AHA/ACC/ AACVPR/AAPA/ABC/ACPM/ADA/AGS/APhA/ASPC/NLA/PCNA guideline on the management of blood cholesterol: Executive summary. Circulation2018.

13 Klein S, Allison DB, Heymsfield SB, et al. Waist circumference and cardiometabolic risk: a consensus statement from shaping
America's health: association for weight management and obesity prevention; NAASO, the obesity Society; the American Society for nutrition; and the American diabetes association. Diabetes Care 2007;30:1647-52.

14 WHO. Who / waist circumference and waist-hip ratio. who, 2008. Available: http://www.who.int/nutrition/publications/obesity/WHO report_waistcircumference_and_waisthip_ratio/en/ [Accessed 12 Dec 2018].

15 Arija V, Villalobos F, Pedret R, et al. Effectiveness of a physical activity program on cardiovascular disease risk in adult primary health-care users: the "Pas-a-Pas" community intervention trial. BMC Public Health 2017;17:576.

16 Heath GW, Parra DC, Sarmiento OL, et al. Evidence-Based intervention in physical activity: lessons from around the world. Lancet 2012;380:272-81.

17 Marcus BH, Williams DM, Dubbert PM, Dubbert Patricia $\mathrm{M}$, et al. Physical activity intervention studies. Circulation 2006;114:2739-52.

18 Dowell D, Farley TA. Prevention of non-communicable diseases in New York City. The Lancet 2012;380:1787-9.

19 WHO. Global action plan on physical activity 2018-2030, 2018. Available: http://apps.who.int/iris/bitstream/handle/10665/ 272722/9789241514187-eng.pdf?ua=1 [Accessed 16 Oct 2018].

20 WHO. Interventions on diet and physical activity: what works : summary report, 2009. Available: http://www.ncbi.nlm.nih.gov/ books/NBK177205/ [Accessed 9 Oct 2018].

21 Proper KI, Staal BJ, Hildebrandt VH, et al. Effectiveness of physical activity programs at worksites with respect to work-related outcomes. Scand J Work Environ Health 2002;28:75-84.

22 Proper KI, Koning M, van der Beek AJ, et al. The effectiveness of worksite physical activity programs on physical activity, physical fitness, and health. Clin J Sport Med 2003;13:106-17.

23 Engbers LH, van Poppel MNM, Chin A Paw MJM, et al. Worksite health promotion programs with environmental changes: a systematic review. Am J Prev Med 2005;29:61-70.

24 Chau JY, der Ploeg HPvan, van Uffelen JGZ, et al. Are workplace interventions to reduce sitting effective? A systematic review. Prev Med 2010;51:352-6.

25 Conn VS, Hafdahl AR, Cooper PS, et al. Meta-Analysis of workplace physical activity interventions. Am J Prev Med 2009;37:330-9.

26 Bergman F, Wahlström V, Stomby A, et al. Treadmill workstations in office workers who are overweight or obese: a randomised controlled trial. Lancet Public Health 2018;3:e523-35.

27 Guazzi M, Faggiano P, Mureddu GF, et al. Worksite health and wellness in the European Union. Prog Cardiovasc Dis 2014:56:508-14

28 Guthold R, Stevens GA, Riley LM, et al. Worldwide trends in insufficient physical activity from 2001 to 2016: a pooled analysis of 358 population-based surveys with 1.9 million participants. Lancet Glob Health 2018;6:e1077-86.

29 WHO. United Arab Emirates health profile 2015, 2016. Available: http://applications.emro.who.int/dsaf/EMROPUB_2016_EN_19276. pdf

30 United Arab Emirates, vision 2021, 2010. Available: https://www. vision2021.ae/docs/default-source/default-document-library/ uae_vision-arabic.pdf?sfvrsn=b09a06a6_6 [Accessed 6 May 2019].

31 Finucane FM, Sharp SJ, Purslow LR, et al. The effects of aerobic exercise on metabolic risk, insulin sensitivity and intrahepatic lipid in healthy older people from the Hertfordshire cohort study: a randomised controlled trial. Diabetologia 2010;53:624-31.

32 Klika B, Jordan C. High-Intensity circuit training using body weight: maximum results with minimal investment. ACSM's Health \& Fitness Journal 2013;17:8-13.

33 Irving BA, Davis CK, Brock DW, et al. Effect of exercise training intensity on abdominal visceral fat and body composition. Med Sci Sports Exerc 2008;40:1863-72.

34 Molmen-Hansen HE, Stolen T, Tjonna AE, et al. Aerobic interval training reduces blood pressure and improves myocardial function in hypertensive patients. Eur J Prev Cardiol 2012;19:151-60.

35 Tomeleri CM, Ribeiro AS, Souza MF, et al. Resistance training improves inflammatory level, lipid and glycemic profiles in obese older women: a randomized controlled trial. Exp Gerontol 2016;84:80-7.

36 Tomeleri CM, Souza MF, Burini RC, et al. Resistance training reduces metabolic syndrome and inflammatory markers in older women: a randomized controlled trial. $J$ Diabetes 2018;10:328-37.

37 Tjønna AE, Ramos JS, Pressler A, et al. EX-MET study: exercise in prevention on of metabolic syndrome - a randomized multicenter trial: rational and design. BMC Public Health 2018;18:437. 
38 Kim Y, Wijndaele K, Sharp SJ, et al. Specific physical activities, sedentary behaviours and sleep as long-term predictors of accelerometer-measured physical activity in 91,648 adults: a prospective cohort study. Int J Behav Nutr Phys Act 2019;16:41.

39 Doherty A, Jackson D, Hammerla N, et al. Large scale population assessment of physical activity using wrist worn Accelerometers: the UK Biobank study. PLoS One 2017;12:e0169649.

40 International Physical Activity Questionnaire. IPAQ scoring protocol International Physical Activity Questionnaire, 2005. Available: https:// sites.google.com/site/theipaq/scoring-protocol [Accessed 27 Jan 2019].

41 Topp CW, Østergaard SD, Søndergaard S, et al. The WHO-5 well-being index: a systematic review of the literature. Psychother Psychosom 2015;84:167-76.
42 Cheikh Ismail L, Osaili TM, Mohamad MN, et al. Eating habits and lifestyle during COVID-19 Lockdown in the United Arab Emirates: a cross-sectional study. Nutrients 2020;12:3314.

43 Hopkins WG, Batterham AM, Rates E. Error rates, decisive outcomes and publication bias with several Inferential methods. Sports Med 2016;46:1563-73.

44 Flynn R, Rotter T, Hartfield D, et al. A realist evaluation to identify contexts and mechanisms that enabled and hindered implementation and had an effect on sustainability of a lean intervention in pediatric healthcare. BMC Health Serv Res 2019;19:912.

45 Whelton PK, Carey RM, Aronow WS, et al. 2017 ACC/AHA/AAPA ABC/ACPM/AGS/APhA/ASH/ASPC/NMA/PCNA guideline for the prevention, detection, evaluation, and management of high blood pressure in adults. J Am Coll Cardiol 2018;71:e127-248. 